\title{
Radiological differentiation of phaeochromocytoma from other malignant adrenal masses: importance of wash-in characteristics on multiphase CECT
}

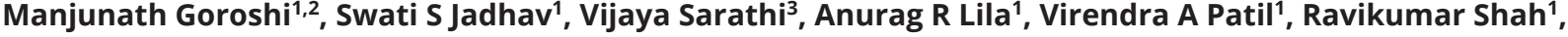 \\ Priya Hira ${ }^{4}$, Rajaram Sharma ${ }^{4}$, Shettepppa Goroshi ${ }^{5}$, Gwendolyn Fernandes ${ }^{6}$, Amey Rojekar ${ }^{6}$, Abhay Dalvi ${ }^{7}$, \\ Ganesh Bakshi ${ }^{8}$, Gagan Prakash ${ }^{8}$, Nalini S Shah ${ }^{1}$ and Tushar R Bandgar ${ }^{1}$
}

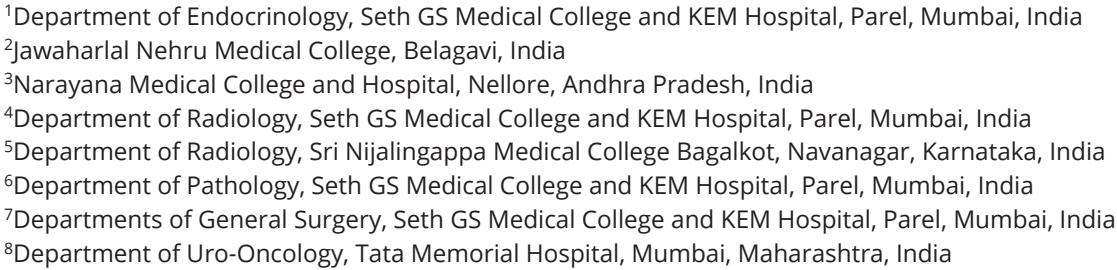

Correspondence should be addressed to S S Jadhav: drswatijadhav1980@gmail.com

\begin{abstract}
Rationale and introduction: To evaluate the computerised tomography (CT) characteristics of phaeochromocytoma (PCC) that differentiate them from other non-benign adrenal masses such as adrenocortical carcinoma (ACC), primary adrenal lymphoma (PAL) and adrenal metastases (AM).

Methods: This retrospective study was conducted at a tertiary health care institute from Western India. Patients presented between January 2013 and August 2016 with histological diagnosis of PCC or other non-benign adrenal mass having adequate reviewable imaging data comprising all four CECT phases were included. Results: The study cohort consisted of 72 adrenal masses from 66 patients (33 PCC, 22 ACC, 4 PAL, 13 AM). Unlike other masses, majority of PCC (25/33) showed peak enhancement in early arterial phase (EAP). PCC had significantly higher attenuation in EAP and early venous phase (EVP), and higher calculated percentage arterial enhancement (PAE) and percentage venous enhancement (PVE) than other adrenal masses $(P<0.001)$. For diagnosis of PCC with $100 \%$ specificity, PAE value $\geq 100 \%$ and EAP attenuation $\geq 100 \mathrm{HU}$ had 78.8 and $63.6 \%$ sensitivity respectively. ACC were significantly larger in size as compared to PCC and metastasis. The adreniform shape was exclusively found in PAL (two out of four) and AM (4 out of 13). None of the enhancement, wash-in or washout characteristics were discriminatory among ACC, PAL and AM. Conclusion: Peak enhancement in EAP, PAE value $\geq 100 \%$ and EAP attenuation $\geq 100 \mathrm{HU}$ differentiate PCC from other malignant adrenal masses with high specificity.
\end{abstract}

\section{Key Words}

- multiphase CECT

- phaeochromocytoma

- percentage arterial enhancement

- peak arterial enhancement https://ec.bioscientifica.com

https://doi.org/10.1530/EC-19-0198 (c) 2019 The authors Published by Bioscientifica Ltd

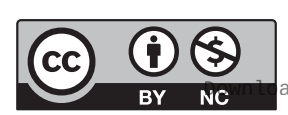

This work is licensed under a Creative Commons Attribution-NonCommercial 4.0 International License. ded from Bioscientifica.com at 04/26/2023 09:13:57AM
Endocrine Connections (2019) 8, 898-905 


\section{Introduction}

There is a recent rise in detection of adrenal incidentalomas (3-10\%) with increasing use of radiological investigations. Benign adenomas are the most common lesions, accounting for $80 \%$ of adrenal incidentalomas, while adrenocortical carcinoma (ACC, $8 \%$ ), phaeochromocytoma (PCC, 7\%) and adrenal metastasis (AM, 5\%) are other less common masses (1). Most crucial step in management of an adrenal incidentaloma is to differentiate a benign 'leave-alone' lesion, from the one that warrants further management. Hence, radiological differentiation of benign adenomas from other non-adenoma adrenal masses has been extensively studied (2). Phaeochromocytoma, a tumour that arises from chromaffin cells of adrenal medulla, constitutes an important non-adenoma adrenal mass. It is usually a benign tumour but can rarely be malignant $(<10 \%)$. Although biochemical evaluation for catecholamine excess state can diagnose most of the PCCs, identification of specific and distinguishing radiological characteristics may provide useful clues to the clinicians. Because of obvious clinical implications, radiological differentiation of PCC from benign adenomas has been evaluated in several computed tomography (CT)-based studies (2). However, studies on radiological differentiation of PCC from other common malignant adrenal masses such as ACC, AM and primary adrenal lymphoma (PAL) are limited. Certain magnetic resonance imaging (MRI)- and MR spectroscopybased characteristics have been shown to distinguish PCC from other malignant adrenal masses (3). In this regard, data on utility of CT-based characteristics, a more commonly used modality for abdominal imaging, are limited.

Characteristics on triple phase contrast-enhanced CT (unenhanced (UP), $60 \mathrm{~s}$ early venous (EVP) and 10-15 min delayed venous phase (DVP)) have not been found useful to differentiate PCC from other nonadenoma masses (4). Although hypervascular nature with avid enhancement during early arterial phase (EAP) is a known feature of PCC, systematic evaluation of arterial phase parameters to distinguish PCC from other malignant adrenal masses has not been reported (5). Hence, we aimed to study the wash-in characteristics that can distinguish PCC from other malignant adrenal masses in a retrospective cohort of patients with adrenal masses who had undergone multiphase CECT of adrenals.

\section{Materials and methods}

\section{Patients}

The study was conducted at a tertiary health care institute from Western India. The study was approved by the Seth GSMC and KEM Hospital Ethics Committee and considering retrospective nature of the study, waiver for patients' consent was granted. We did a retrospective chart analysis of patients presented to our department between January 2013 and August 2016 with adrenal mass. Inclusion criteria were patients with available histological diagnosis of PCC or other non-benign adrenal mass having adequate reviewable imaging data comprising all four CECT phases that is UP, EAP, EVP and DVP. Final study cohort consisted of 72 adrenal masses in 66 patients (six patients had bilateral adrenal masses). These included 33 PCC, 22 ACC, 4 PAL and 13 AM (Fig. 1).

Baseline demographic features like age and preoperative hormonal profile were recorded for each patient. PCC were classified as metanephrine-secreting (increased plasma free metanephrine with/without elevation of plasma free normetanephrine), normetanephrinesecreting (elevated plasma free normetanephrine but normal plasma free metanephrine) or non-secretory (normal plasma free metanephrines). ACC was considered

Patients with adrenal masses diagnosed between January 2013 and August 2016 at our centre $(n=159)$

Histopathological diagnosis available $(n=80)$

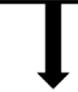

Phaeochromocytoma or malignant lesions $(n=74)$

Adequately reviewable four phase CECT available $(n=66)$
66 patients
30 phaeochromocytoma (bilateral in 3 )
22 adrenocortical carcinoma
4 primary adrenal lymphoma
10 adrenal metastasis (bilateral in 3 )

Figure 1

Flow chart showing assessment of adrenal masses for inclusion in the study.

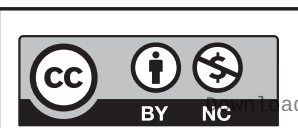

This work is licensed under a Creative Commons Attribution-NonCommercial 4.0 International License. ded from Bioscientifica.com at 04/26/2023 09:13:57AM via free access 
as cortisol secreting, if overnight dexamethasone supressed serum cortisol was $\geq 1.8 \mu \mathrm{g} / \mathrm{dL}$. Diagnosis of patients with AM was based on primary tumour histopathology and/or adrenal biopsy.

\section{CT protocol}

Imaging of the abdomen was performed on a 64-slice multidetector CT system (Brilliance 64, Philips Healthcare) using standardised protocol. Patients were placed in supine position, with arms pulled caudally. The scanning protocol consisted of four identical helical scans obtained in an automated, predetermined and timed sequence. Scanning parameters were $120 \mathrm{kVp}$, with automatic exposure control (range, 140-220 mA), 0.75 seconds rotation time, pitch of 0.797 and a 0.625 $\mathrm{mm}$ detector configuration, with beam width of $40 \mathrm{~mm}$. First phase was obtained at baseline (UP). $100 \mathrm{~mL}$ of iodinated contrast material (Iohexol: Omnipaque 300, GE Healthcare) was injected in the antecubital vein through a preplaced 18-gauge cannula, at the rate of $3 \mathrm{~mL} / \mathrm{s}$ and was followed by a saline flush. The second phase (EAP) was obtained after $20 \mathrm{~s}$ of contrast administration. Third phase (EVP) and fourth phase (DVP) were obtained at $1 \mathrm{~min}$ and 15 min respectively. All scans obtained were stored on mass storage device (Seagate, Cupertino, CA, USA) and retrieved by attaching mass storage devices to picture archiving and communication system (PACS) as direct storage device. Images were retrieved by transferring them back to the PACS. Images were reconstructed in standard radiology workstation consisting of trans-axial, sagittal and coronal projections.

\section{Imaging analysis}

Two radiologists (with 21 years and 7 years of experience) who were blinded to histopathological diagnosis reported the CT images on workstation. Morphological parameters like size (maximum diameter in axial images), shape (spherical, adreniform or irregular) and presence of necrosis, haemorrhage and calcification were noted. For each phase, attenuation in HU was measured by placing elliptic region of interest (ROI) of minimum $1 \mathrm{~cm}^{2}$, at three separate sections of the mass, and mean of these three values was calculated. ROI was placed on maximally enhancing areas in the EAP and replicated in identical locations for other (UP, EVP and DP) phases. For ROI placement, due care was taken to cover the maximum possible area, after excluding areas of calcification, necrosis, haemorrhage and surrounding fat. Additionally, aortic HU (at the level of renal artery) was measured in EAP. Relative washout percentage $(\mathrm{RWP}=(\mathrm{EVP} \mathrm{HU}-\mathrm{DP}$ $\mathrm{HU}) \times 100 / \mathrm{EVP} \mathrm{HU})$ and absolute washout percentage $($ AWP $=($ EVP HU - DP HU $) \times 100 /($ EVP HU - UP HU $)$ were calculated. 'Peak enhancement phase' was defined as the phase in which enhancement was maximum with attenuation value greater than other phases by $>5 \mathrm{HU}$. Percentage arterial enhancement (PAE) was calculated as a measure of enhancement in EAP as compared to that in UP and was calculated as $\mathrm{PAE}=(\mathrm{EAP} \mathrm{HU}-\mathrm{UP}$ HU) $\times 100 /$ UP HU. Percentage venous enhancement (PVE) was calculated as a measure of enhancement in EVP as compared to UP and was calculated as $\mathrm{PVE}=(\mathrm{EVP} H \mathrm{HU}-\mathrm{UP}$ HU) $\times 100 /$ UP HU.

Statistical analysis was done using SPSS version 23, IBM. Six patients in our cohort had bilateral masses. The statistical analysis was done with 'mass' (rather than patient) as a unit. Any missed data (demographic features and hormonal profile) were addressed using multiple imputation method. Categorical variables (shape, presence/absence of necrosis, calcification and haemorrhage, peak enhancement phase) were expressed in actual numbers or percentages and compared by Kruskal-Wallis test. Continuous variables (age, size, HU in different phases, AWP, RWP, PAE, PVE and aortic HU) were expressed as mean \pm s.D. and were compared using one-way ANOVA. Post hoc test (LSD) was done to study intergroup comparisons.

Inter-rater reliability for shape, presence of necrosis and calcification was assessed by calculating Cohen's Kappa (K) value. $\mathrm{K}$ value more than 0.6 was considered as good level of agreement. Inter-observer variation in measurement of attenuation values was assessed by interclass correlation coefficient (ICC), and a value more than 0.8 was considered as good correlation. Receiver-operating characteristic (ROC) curves were used to determine the cut-off value(s) for different CECT parameters that can best differentiate PCC from other adrenal masses with maximum specificity. $P$ value $<0.05$ was considered as statistically significant.

\section{Results}

The study cohort consisted of 72 adrenal masses from 66 patients (6 patients with bilateral adrenal masses). Mean age of the study population was $41.4 \pm 16.8$ years. Cohen's kappa (k) value for inter-rater reliability among the two radiologists for shape, presence of necrosis and calcification was $0.978(P<0.001,95 \%$ CI $0.95-0.98)$. 
ICC for $\mathrm{HU}$ measurements between the two radiologists was 0.98 ( $P<0.001,95 \%$ CI $0.97-0.98)$. Comparison of morphological, contrast enhancement and washout characteristics of various adrenal masses is shown in Table 1. Age of presentation was not significantly different among the different groups of adrenal masses.

\section{PCC}

Thirty-three PCCs were included in the analysis (three patients had bilateral PCCs). Twenty-two PCCs were normetanephrine-secreting, nine were metanephrinesecreting and two were non-secretory. In UP, the attenuation of PCC was not significantly different from those of other subgroups (Figs 2, 3 and Table 1). Majority of PCCs (25/33) showed peak enhancement in EAP, whereas six PCCs had peak enhancement in EVP and remaining two PCC were equally enhancing (peak) in EAP and EVP (difference being $<5 \mathrm{HU}$ ). None of the masses from other subgroups had peak enhancement in EAP. PCCs had significantly higher attenuation in EAP and EVP, and higher calculated PAE and PVE than other adrenal masses $(P<0.001)$. Among these, arterial phase characteristics had the best AUC in ROC curve analysis to differentiate PCC from other masses (Fig. 4 and Table 2). For diagnosis of PCC with $100 \%$ specificity,
PAE value $\geq 100 \%$ and EAP HU $\geq 100$ had $78.8 \%$ and $63.6 \%$ sensitivity respectively.

Out of seven PCCs which had PAE $<100 \%$, five were metanephrine secreting. Similarly, out of 12 PCCs with EAP HU $<100$, eight were metanephrine secreting. Thus, based on the observation that higher proportion of PCC with PAE $<100 \%$ and EAP HU $<100$ were metanephrine-secreting, sub-group analysis was done to compare CT characteristics of PCC with these secretory subtypes (Table 3). Metanephrine-secreting PCC had significantly lower attenuation in EAP and EVP, lower PAE and PVE as compared to nor-metanephrine-secreting PCC. Additionally, proportion of the PCC with peak enhancement in EAP or EAP HU $\geq 100$ were significantly lower in the metanephrine-secreting PCC group.

\section{ACC, AM and PAL}

Twenty-two ACCs (ten were cortisol secreting), 4 PAL and $13 \mathrm{AM}$ (three patients with bilateral metastases) were included in our study. ACCs were significantly larger in size as compared to PCC and metastasis. The adreniform shape was exclusively found in PAL (two out of four) and AM (4 out of 13). While necrosis was prevalent in almost all the groups of adrenal masses, calcifications were seen in a minority of PCC and ACC. Adrenal masses in each

Table 1 Comparison of morphological, contrast enhancement and washout characteristics of various types of adrenal masses.

\begin{tabular}{l}
\hline \\
\hline Age (years) \\
Morphological characteristics \\
Shape \\
$\quad$ Spherical \\
$\quad$ Adreniform \\
$\quad$ Irregular \\
Size (cm) \\
Necrosis \\
Calcification \\
Haemorrhage \\
Enhancement patterns \\
Unenhanced phase enhancement (HU) \\
Arterial phase enhancement (HU) \\
Early venous phase enhancement (HU) \\
Delayed venous phase enhancement (HU) \\
Absolute percentage washout (\%) \\
Relative percentage washout (\%) \\
Percentage arterial enhancement (\%) \\
Percentage venous enhancement (\%) \\
Aortic enhancement (HU)
\end{tabular}

\begin{tabular}{c} 
PCC $(n=33)$ \\
\hline $38.8 \pm 18.6$ \\
\\
23 \\
0 \\
10 \\
$5.5 \pm 2.4$ \\
32 \\
2 \\
0
\end{tabular}

$\begin{aligned} 37.1 & \pm 6.1 \\ 117.6 & \pm 41.1 \\ 102.7 & \pm 29.4 \\ 76.5 & \pm 42.3 \\ 34.1 & \pm 20.6 \\ 21.2 & \pm 20.5 \\ 227 & \pm 129 \\ 186.6 & \pm 102.4 \\ 285 & \pm 65\end{aligned}$

ACC $(n=22)$

$41.5 \pm 12.3$

$\frac{\text { Lymphoma }(n=4)}{36.4 \pm 18.2}$

M

\begin{tabular}{c}
\hline P value \\
\hline 0.145 \\
\\
$<0.001^{a}$ \\
\\
$<0.001^{b}$ \\
0.42 \\
0.194
\end{tabular}

$38.4+6.02$ $54.8 \pm 11.9$ $70.1 \pm 12.1$ $55.9 \pm 12.5$ $44.2 \pm 23$ $19.6 \pm 12.7$ $43.4 \pm 28.1$ $84.1 \pm 31.5$ $256.9 \pm 85.2$

Post hoc test (LSD).

a $P$ value significant between lymphoma vs PCC $(<0.001)$, ACC $(<0.001)$ and metastases $(0.001)$; ${ }^{b} P$ value significant between $P C C$ vs ACC $(P<0.001)$ and $A C C$ vs metastasis $(P<0.001)$; ${ }^{c} P$ value significant between PCC vs ACC $(P<0.001)$, lymphoma $(<0.001)$ and metastasis $(P<0.001)$; ${ }^{2} P$ value significant between PCC vs ACC $(P<0.001)$, lymphoma $(0.001)$ and metastasis $(P<0.001)$; e $P$ value significant between PCC vs ACC $(P=0.012)$ and metastasis $(P=0.001)$. ACC, adrenal cortical carcinoma; PCC, phaeochromocytoma.

https://ec.bioscientifica.com https://doi.org/10.1530/EC-19-0198 (c) 2019 The authors Published by Bioscientifica Ltd

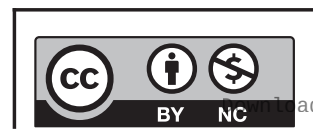

This work is licensed under a Creative Commons Attribution-NonCommercial 4.0 International License. ded from Bioscientifica.com at 04/26/2023 09:13:57AM 

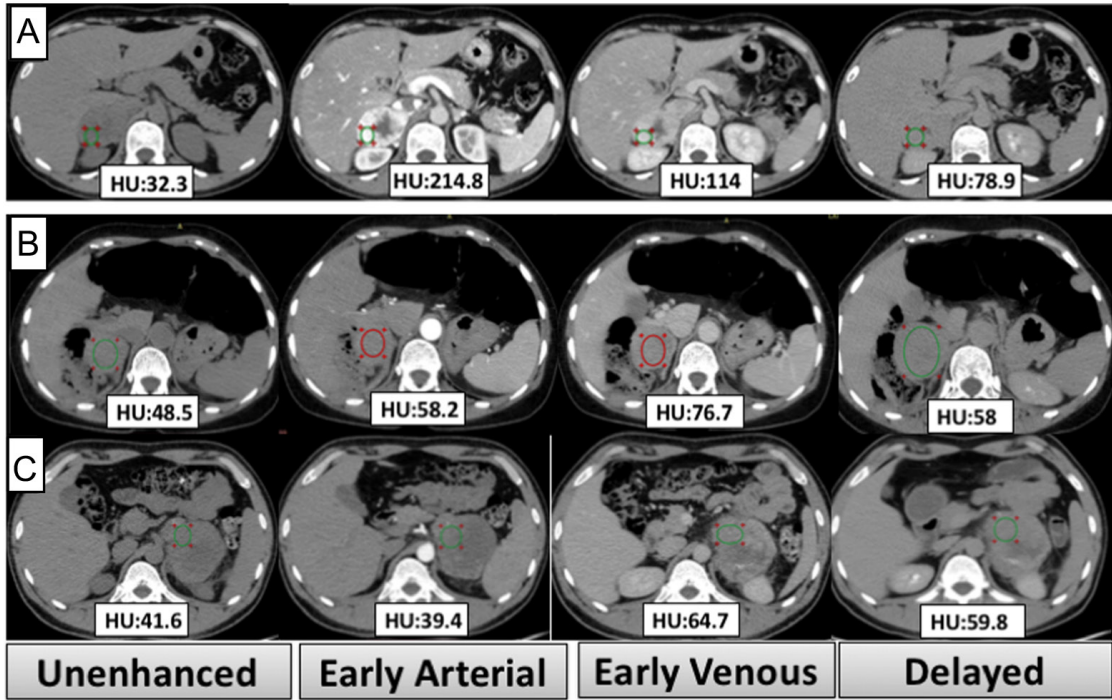

Figure 2

(A, B and C) Axial CECT images with attenuation value mentioned in different phases. (A) Right adrenal phaeochromocytoma (nor-metanephrine secreting) showing peak enhancement in early arterial phase. (B) Right adrenal phaeochromocytoma (metanephrine secreting) showing peak enhancement in early venous phase. (C) Left adrenocortical carcinoma.

of these three subgroups had peak enhancement in EVP and none of the enhancement, wash-in or washout characteristics were discriminatory among these three subgroups (Table 1).

\section{Discussion}

Up to $14 \%$ of adrenal incidentalomas are reported to be PCCs (1). Especially amidst the non-adenoma adrenal masses, PCC form a sizeable proportion of incidentalomas $(\sim 35 \%)$, making its radiological characterisation a pertinent area of investigation. While CECT-based differentiation of PCC from benign adenomas has been extensively studied, there are limited studies on radiological differentiation of PCC from other malignant masses. In the current study, we report multiphase CECT characteristics that differentiate PCC from other malignant adrenal masses like ACC, AM and PAL. Previously, Szolar et al. (4) have reported CT attenuation characteristics on unenhanced, $1 \mathrm{~min}$ and
10 min post contrast scans of 73 adrenal masses which included ACC, PCC, AM and adenomas. They found no significant difference in either mean attenuation $\mathrm{HU}$ values in any of the three phases or washout percentages among PCC, ACC and AM. In contrast, we found that mean attenuation $\mathrm{HU}$ values in EVP were significantly higher in PCC than in ACC and AM. Lesser number of PCC $(n=17)$ and presence of few $(n=2)$ hypervascular metastases in their cohort might have accounted for this difference in findings (4).

In our study, we found that addition of wash-in EAP parameters (PAE value $\geq 100 \%$, peak enhancement in EAP and EAP attenuation $\geq 100 \mathrm{HU}$ ) provided the best diagnostic accuracy to differentiate PCC from other malignant adrenal masses. Although wash-in characteristics of adrenal masses on CECT have been studied previously $(6,7,8,9)$, studies reporting systematic evaluation of diagnostic accuracy of various EAP parameters to differentiate PCC from other adrenal masses have been limited. In a study by Szolar et al., wash-in characteristics of 74 adenomas and
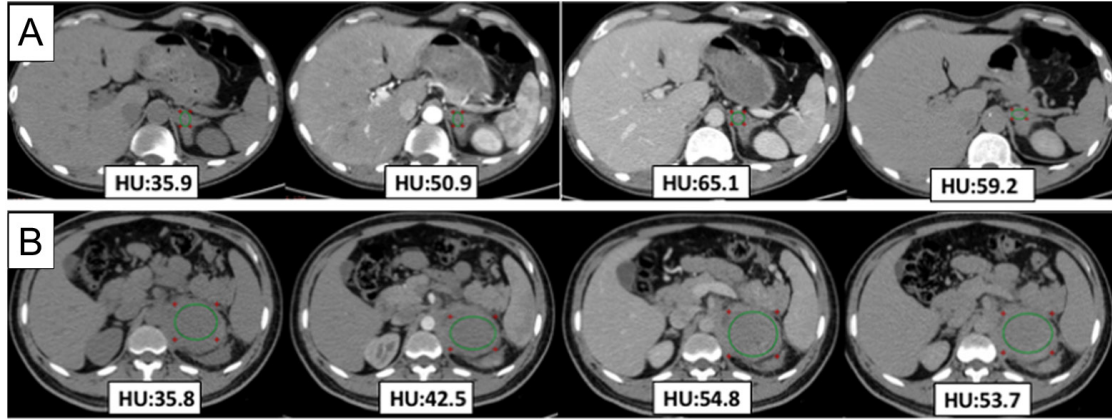

\section{\begin{tabular}{l|l|l|l|l|l|l|} 
Unenhanced Early Arterial Early Venous Delayed \\
\hline
\end{tabular}}

Figure 3

(A and B) Axial images of CECT with attenuation value mentioned in different phases. (A) Left adrenal metastases. (B) Left primary adrenal lymphoma. https://ec.bioscientifica.com https://doi.org/10.1530/EC-19-0198 (c) 2019 The authors Published by Bioscientifica Ltd

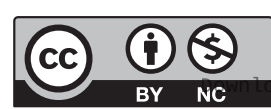

This work is licensed under a Creative Commons Attribution-NonCommercial 4.0 International License. ded from Bioscientifica.com at 04/26/2023 09:13:57AM 


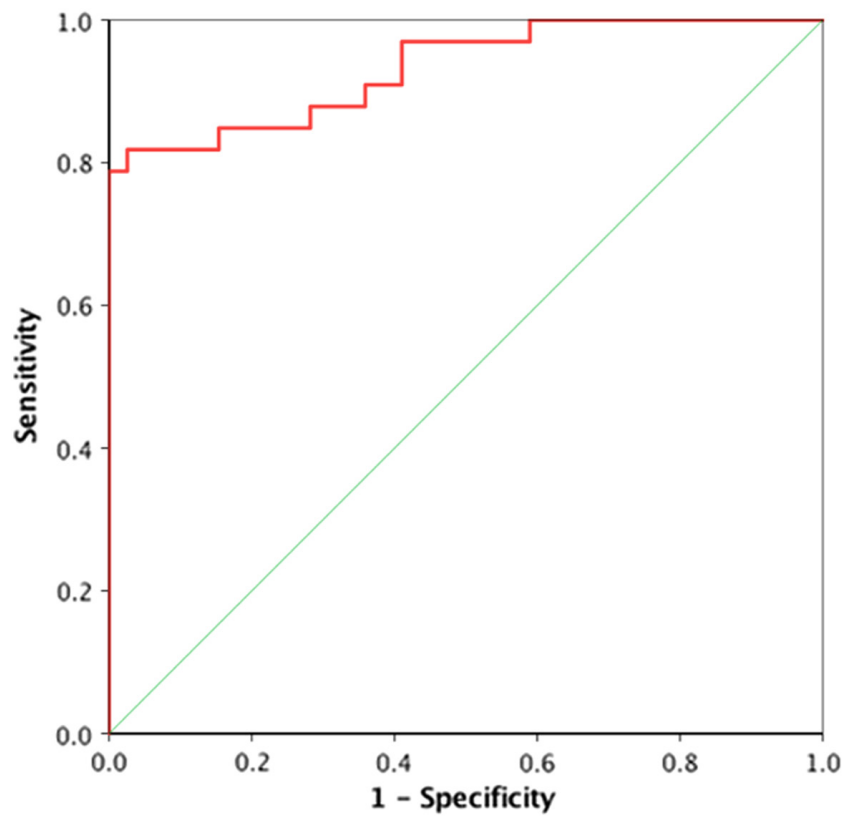

Figure 4

The receiver-operating curve (ROC) analysis for different parameters for discriminating phaeochromocytoma from other malignant adrenal masses.

61 nonadenomas were studied (6). They found the adenomas to have significantly higher attenuation at $30 \mathrm{~s}$ and higher PAE (referred in their paper as 'percentage increase in attenuation') than nonadenomas. However, their non-adenoma masses mostly consisted of metastases $(n=47)$ and few PCC $(n=6$, 30 s scan available for 2 PCC only) and no comparison was made amidst the various non-adenoma masses. Use of PVE as a wash-in parameter was reported by Foti et al. in their study of 107 adrenal masses (56 adenomas and 51 metastasis) (9). While they found no significant difference in PVE (referred in their study as 'relative percentage wash-in ratio from the unenhanced to the portal venous phase') between adenomas and metastasis, there were no PCCs in their cohort. 'Wash-in attenuation' (calculated as the difference between $35 \mathrm{~s}$ enhanced attenuation and

Table 2 Diagnostic performance of various imaging parameters represented by area under the curve calculated using receptor-operating characteristic curves.
Parameter

Percentage arterial enhancement (\%) Early arterial phase enhancement (HU)

Early venous phase enhancement (HU) Percentage venous enhancement (\%) Delayed phase enhancement (HU) Relative percentage washout (\%) Absolute percentage washout (\%)

\begin{tabular}{|c|c|}
\hline AUC & $95 \% \mathrm{Cl}$ \\
\hline 0.93 & $0.87-0.99$ \\
\hline 0.91 & $0.86-0.99$ \\
\hline 0.86 & $0.77-0.95$ \\
\hline 0.80 & $0.69-0.91$ \\
\hline 0.81 & $0.71-0.91$ \\
\hline 0.49 & $0.41-0.68$ \\
\hline 0.67 & $0.55-0.80$ \\
\hline
\end{tabular}

https://ec.bioscientifica.com https://doi.org/10.1530/EC-19-0198 (c) 2019 The authors Published by Bioscientifica Ltd unenhanced attenuation) and 'phase of achieving peak enhancement' are the other wash-in parameters reported in studies mainly comparing adenomas with nonadenomas $(7,8)$. However, we believe that our results are not directly comparable with any of these previous studies reporting wash-in characteristics of adrenal masses, due to basic differences in composition of the cohorts (with no adenomas in our cohort and few PCC in previous studies).

We found interesting differences in attenuation parameters between biochemical sub-types of PCC, with normetanephrine-secreting PCC having higher attenuation profile than metanephrine-secreting PCCs. To the best of our knowledge, this has not been reported previously. This is most probably due to implication of hypoxia pathway defects (Cluster 1 genes: VHL and SDHx) in the pathogenesis of former than the latter (Cluster 2: RET, NF1, TMEM127) (10). Hypervascular nature of PCC is also the most probable reason for their higher arterial enhancement than other malignant masses.

Although not an aim of our study, differentiating PCC from benign adenomas is important from the clinical standpoint. Since both adenomas and PCC can have good as well as poor contrast washout patterns $(2,11)$, it would be informative to test the performance of wash-in EAP parameters in differentiating PCC from adenomas. In this regard, a study by Northcutt et al. compared EAP (25s) and EVP (1 min) enhancement of 12 PCC with 41 adenomas (7). They also found EAP parameters (absolute arterial enhancement $>110 \mathrm{HU}$ and peak enhancement in EAP) as specific for PCC, distinguishing them from adenomas. While PAE and PVE of adenomas vs PCC have not been studied adequately, EAP attenuation values of adenomas have been consistently shown to be significantly lower than that of PCC and can provide useful clues $(7,12,13)$.

Our findings can have some implications in the evaluation of incidental adrenal masses. Arterial phase wash-in characteristics can be readily obtained from the routinely performed triple-phase (EAP, EVP and 3-min DVP) CECT abdomen scans, the scan in which an adrenal incidentaloma is often diagnosed first. In case of an incidentaloma, documenting hypervascular nature using wash-in characteristics differentiates PCC from ACC and other malignant masses, as demonstrated in our study, as well as from adrenal adenoma as demonstrated by previous studies $(7,12,13)$. This may reduce the need for a repeat adrenal focussed multiphase CECT imaging and may also help to minimise the laboratory evaluation by choosing plasma-free metanephrines, particularly

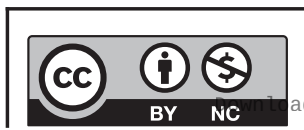

This work is licensed under a Creative Commons Attribution-NonCommercial 4.0 International License. ded from Bioscientifica.com at 04/26/2023 09:13:57AM 
Table 3 Comparison of computed tomography characteristics of metanephrine-secreting and normetanephrine-secreting phaeochromocytoma.

\begin{tabular}{l}
\hline \\
\hline Number of tumours with maximum \\
enhancement in arterial phase (\%) \\
Number of tumours with arterial phase \\
enhancement $>100 \mathrm{HU}(\%)$ \\
Size $(\mathrm{cm})$ \\
Unenhanced phase enhancement (HU) \\
Arterial phase enhancement (HU) \\
Early venous phase enhancement (HU) \\
Delayed venous phase enhancement (HU) \\
Percentage arterial enhancement (\%)
\end{tabular}

\begin{tabular}{c}
\hline Normetanephrine secreting $(n=22)$ \\
\hline $20(90.9 \%)$ \\
$18(81.8 \%)$ \\
$5.6 \pm 2.2$ \\
$37.2 \pm 6.6$ \\
$136.6 \pm 33.6$ \\
$115 \pm 26.4$ \\
$86.6 \pm 48.3$ \\
$279.4 \pm 114.8$ \\
\hline
\end{tabular}

\begin{tabular}{c}
\hline Metanephrine secreting $(n=9)$ \\
\hline $5(55.5 \%)$ \\
$1(11 \%)$ \\
$5.8 \pm 3.2$ \\
$37.5 \pm 5.6$ \\
$78.4 \pm 24.8$ \\
$75.6 \pm 18.2$ \\
$56 \pm 14.1$ \\
$117.1 \pm 90$ \\
\hline
\end{tabular}

\begin{tabular}{c}
\hline P value \\
\hline 0.047 \\
\\
$<0.001$ \\
\\
0.83 \\
0.907 \\
$<0.001$ \\
$<0.001$ \\
0.07 \\
0.001
\end{tabular}

HU, Hounsfield units.

plasma free normetanephrine as the most pertinent initial biochemical test.

Technical factors related to contrast administration, cardiovascular status of patients, and true biological tumoural vascular characteristics are the few important factors that can influence arterial enhancement pattern of a tissue $(14,15)$. Since this is a single-centre study performed over a relatively shorter duration, the technical aspects like type, amount and rate of contrast agent injection have largely remained uniform for patients included in the study. The fact that the aortic HU was similar among different tumour groups rules out the confounding effect of cardiovascular status on enhancement patterns (Table 1). Hence, it is likely that the difference in enhancement patterns in our study reflects the biological difference in underlying tumoural vascular characteristics. Considering robust reference of histopathology for diagnosis and reporting of images in a blinded fashion (to minimise the observer error) were other strengths of our study.

By attempting to differentiate PCC from other malignant adrenal masses and to radiologically characterise biochemical sub-types of PCC, we have studied an area less addressed in previous CECT studies on PCCs. However, exclusion of benign masses limits the wide clinical applicability of our results. We have included consecutive patients satisfying the inclusion criteria (to minimise the selection bias); however, due to the retrospective nature of the study, a large proportion of patients with adrenal masses were excluded due to lack of four phase adrenal imaging and/or histopathology (Fig. 1) which might have added significant selection bias. Small sample size is another major limitation of our study. Additionally, few hypervascular adrenal masses such as metastasis from renal-cell carcinoma or hepatocellular carcinoma with potential to yield higher PAE values were not part of our cohort. Hence, we propose the need of larger, multi-centric, prospective studies with wider spectrum of benign as well as malignant masses which helps to confirm and generalise the study findings.

To conclude, we present a report on multiphase CECT-based characterisation of PCC and found that certain arterial phase-based wash-in parameters like PAE value $\geq 100 \%$, peak enhancement in EAP and EAP attenuation $\geq 100 \mathrm{HU}$ can differentiate PCC from other malignant adrenal masses with high specificity.

\section{Declaration of interest}

The authors declare that there is no conflict of interest that could be perceived as prejudicing the impartiality of the research reported.

\section{Funding}

This work did not receive any specific grant from any funding agency in the public, commercial, or not-for-profit sector.

\section{References}

1 Fassnacht M, Arlt W, Bancos I, Dralle H, Newell-Price J, Sahdev A, Tabarin A, Terzolo M, Tsagarakis S \& Dekkers OM. Management of adrenal incidentalomas: European Society of Endocrinology Clinical Practice Guideline in collaboration with the European Network for the Study of Adrenal Tumors. European Journal of Endocrinology 2016 175 G1-G34. (https://doi.org/10.1530/EJE-16-0467)

2 Dinnes J, Bancos I, Di Ruffano LF, Chortis V, Davenport C, Bayliss S, Sahdev A, Guest P, Fassnacht M, Deeks JJ, et al. Management of Endocrine Disease: imaging for the diagnosis of malignancy in incidentally discovered adrenal masses: a systematic review and meta-analysis. European Journal of Endocrinology 2016175 R51-R64. (https://doi.org/10.1530/EJE-16-0461)

3 Faria JF, Goldman SM, Szejnfeld J, Melo H, Kater C, Kenney P, Huayllas MP, Demarchi G, Francisco VV, Andreoni C, et al. Adrenal masses: characterization with in vivo proton MR spectroscopy - initial experience. Radiology 2007245 788-797. (https://doi. org/10.1148/radiol.2453061854)

4 Szolar DH, Korobkin M, Reittner P, Berghold A, Bauernhofer T, Trummer H, Schoellnast H, Preidler KW \& Samonigg H. 
Adrenocortical carcinomas and adrenal pheochromocytomas: mass and enhancement loss evaluation at delayed contrast-enhanced CT. Radiology 2005234 479-485. (https://doi.org/10.1148/ radiol.2342031876)

5 Blake MA, Kalra MK, Maher MM, Sahani DV, Sweeney AT, Mueller PR, Hahn PF \& Boland GW. Pheochromocytoma: an imaging chameleon. RadioGraphics 200424 (Supplement 1) S87-S99. (https:// doi.org/10.1148/rg.24si045506)

6 Szolar DH \& Kammerhuber FH. Adrenal adenomas and nonadenomas: assessment of washout at delayed contrast-enhanced CT. Radiology 1998207 369-375. (https://doi.org/10.1148/radiology.207.2.9577483)

7 Northcutt BG, Raman SP, Long C, Oshmyansky AR, Siegelman SS, Fishman EK \& Johnson PT. MDCT of adrenal masses: can dualphase enhancement patterns be used to differentiate adenoma and pheochromocytoma? American Journal of Roentgenology 2013201 834-839. (https://doi.org/10.2214/AJR.12.9753)

8 Kamiyama T, Fukukura Y, Yoneyama T, Takumi K \& Nakajo M. Distinguishing adrenal adenomas from nonadenomas: combined use of diagnostic parameters of unenhanced and short 5-minute dynamic enhanced CT protocol. Radiology 2009250 474-481. (https://doi.org/10.1148/radiol.2502080302)

9 Foti G, Faccioli N, Manfredi R, Mantovani W \& Mucelli RP. Evaluation of relative wash-in ratio of adrenal lesions at early biphasic CT. American Journal of Roentgenology 2010194 1484-1491. (https://doi.org/10.2214/AJR.09.3636)
10 Favier J \& Gimenez-Roqueplo AP. Pheochromocytomas: the (pseudo)hypoxia hypothesis. Best Practice and Research: Clinical Endocrinology and Metabolism 201024 957-968. (https://doi.org/10.1016/j. beem.2010.10.004)

11 Sangwaiya MJ, Boland GW, Cronin CG, Blake MA, Halpern EF \& Hahn PF. Incidental adrenal lesions: accuracy of characterization with contrast-enhanced washout multidetector CT - 10-minute delayed imaging protocol revisited in a large patient cohort. Radiology 2010256 504-510. (https://doi.org/10.1148/ radiol.10091386)

12 Zhang GMY, Shi B, Sun H, Jin ZY \& Xue HD. Differentiating pheochromocytoma from lipid-poor adrenocortical adenoma by CT texture analysis: feasibility study. Abdominal Radiology $2017 \mathbf{4 2}$ 2305-2313. (https://doi.10.1007/s00261-017-1118-3)

13 Yao R, Hu W, Qian M, Zhang L \& Zhao Y. Correlation between CT image presentations and biochemical indexes in adrenal adenomas and pheochromocytomas. Nan Fang Yi Ke Da Xue Xue Bao 201535 1792-1796.

14 O'Connor JP, Tofts PS, Miles KA, Parkes LM, Thompson G \& Jackson A. Dynamic contrast-enhanced imaging techniques: CT and MRI. British Journal of Radiology 201184 S112-S120. (https://doi. org/10.1259/bjr/55166688)

15 Bae KT. Intravenous contrast medium administration and scan timing at CT: considerations and approaches. Radiology 2010256 32-61. (https://doi.org/10.1148/radiol.10090908)

Received in final form 20 May 2019

Accepted 5 June 2019

Accepted Preprint published online 6 June 2019
This work is licensed under a Creative Commons Attribution-NonCommercial 4.0 International License. ded from Bioscientifica.com at 04/26/2023 09:13:57AM 